\title{
Opioid Overdose-Related Emergency Department Visits and Accidental Deaths during the COVID-19 Pandemic
}

\author{
Luke N. Rodda $(\mathbb{D} \cdot$ Kelsa L. West • Kathy T. LeSaint
}

Accepted: 11 September 2020 /Published online: 28 September 2020

(C) The New York Academy of Medicine 2020

\section{Introduction}

The world became aware of a novel coronavirus disease (COVID-19) in late December 2019, caused by severe acute respiratory syndrome coronavirus 2 (SARS-CoV2). COVID-19 presents a heightened threat to human life with its ability to spread efficiently and at a high mortality rate to those with medical comorbidities [1,2]. Within months, the virus had spread rapidly from its origin in China to parts of Europe, namely Italy and the UK, and to the United States (US). Towards the end of March, the USA had the most cases worldwide [3].

Many US states, counties, and cities enacted travel restrictions and promoted social distancing in order to combat human-to-human spread of COVID-19. In mid-March, San Francisco led the country in enacting these safety measures, and a

L. N. Rodda · K. L. West

Forensic Laboratory Division, Office of the Chief Medical

Examiner, San Francisco, CA, USA

L. N. Rodda $(\bowtie)$

Department of Laboratory Medicine, University of California, San Francisco, San Francisco, CA, USA

e-mail: luke.rodda@ucsf.edu month later, nearly all of mainland US was under similar restrictions. Early prevention measures included limitations on the use of city-owned facilities, followed by a restriction on public gatherings, and on March 16th, a complete shelter-in-place mandate was ordered [4]. Surrounding San Francisco Bay Area, counties enacted similar measures contemporaneously, and California mandated a statewide shelter-in-home (akin to shelter-in-place) restriction on March 19th [5].

Concomitant to the COVID-19 pandemic, San Francisco has been experiencing an opioid epidemic throughout recent years, starting predominantly in 2016, and largely due to the significant increase of fentanyl availability and, often contemporaneous, use with heroin $[6,7]$. News outlets, harm reduction agencies, and professional health societies have since shared

\section{K. T. LeSaint}

Department of Emergency Medicine, University of California, San Francisco, San Francisco, CA, USA

\section{K. T. LeSaint}

California Poison Control System, San Francisco Division, San Francisco, CA, USA 
concerns of increased overdose incidents during periods of social distancing $[8,9]$.

In this study, we describe the prevalence and characteristics of individuals in San Francisco who experienced significant consequences following opioid use, specifically those resulting in emergency department visits and accidental death, in the time of social distancing during the COVID-19 pandemic.

\section{Methods}

Patient cases from January 1 through April 18, 2020, were included to capture the immediate time period of mid-March representing before and after social distancing mandates were enacted. Descriptive statistics were used to characterize the cohort of patients. Categorical variables are described via absolute values and percentages.

\section{Opioid Overdose-Related Emergency Room Presentations}

Together, the emergency departments at the University of California, San Francisco (UCSF) and its affiliated Zuckerberg San Francisco General Hospital \& Trauma Center (ZSFG) serve the largest proportion of patients in the City, each providing care to over 40,000 patients per year. The electronic medical records at both sites were queried for patient chief complaints and final diagnoses of "overdose." Of these, cases were excluded if neither the chief complaint nor the final diagnosis was opiate, opioid, heroin, and/or fentanyl related. Patient-related demographic information such as date of emergency room presentation, age, and sex were collected in addition to the underlying chief complaint.

\section{Opioid Overdose-Related Fatalities}

All accidental, homicide, suicide, suspicious, undetermined, and certain natural deaths in San Francisco are investigated by the Office of the Chief Medical Examiner (OCME). During the study period, all available forensic investigation, medical and scientific findings from accidental overdose cases were captured. For the purposes of this study, demographic and toxicological information were collected on each case. Using a previously published method, accidental overdose deaths with fentanyl, norfentanyl, 6-monoacetylmorphine (6-
MAM, heroin's primary metabolite), morphine, and/or codeine detected in blood were captured to best determine their relevance in causation of drug toxicity [10]. Contribution of heroin to death was assessed following the model described by Stam et al. [11]. Deaths caused by other drugs were not considered in this study.

\section{Results}

\section{Demographics}

Males made up four out of five cases in both emergency department presentations and fatal overdoses (Fig. 1). Younger age groups made the majority of emergency presentations while, comparatively, there were more deaths in elderly populations.

\section{Survived Overdoses}

During the study period, 365 emergency department patients at UCSF and ZSFG were seen with the chief complaint or final diagnosis of "overdose." Of these, 176 visits were either not opioid-related (e.g., overdose related to acetaminophen) or not clearly opioid-related (e.g., final diagnosis was pneumonia) and, therefore, were excluded. A total of 189 patients were seen for opioid-related overdose and were included in the primary analysis. Seventy-eight percent were male with a median age of 37 years (IQR 30-49). There were no in-hospital deaths in this cohort. Substances consumed by patients included fentanyl $(N=31,16 \%)$, heroin $(N=2,1 \%)$, and non-specified opioid $(N=153,81 \%)$. Following the shelter-in-place enactment period to April 18, UCSF and ZSFG emergency departments saw approximately 2.5 patients per day with opioid overdose, compared with 1.4 patients per day prior to this period (Fig. 2).

\section{Fatal Overdoses}

Of the 459 deaths received and accepted by the OCME in the first 109 days of the year in 2020, 121 were opioid-related accidental overdoses. Eighty-two percent were male. Age was unknown in three individuals. The median age for 118 patients with accidental death was 45 years (IQR 35-56). Of accidental overdose death, 95 $(78 \%)$ were related to fentanyl, $13(11 \%)$ related to heroin, and $13(11 \%)$ related to fentanyl and heroin in 

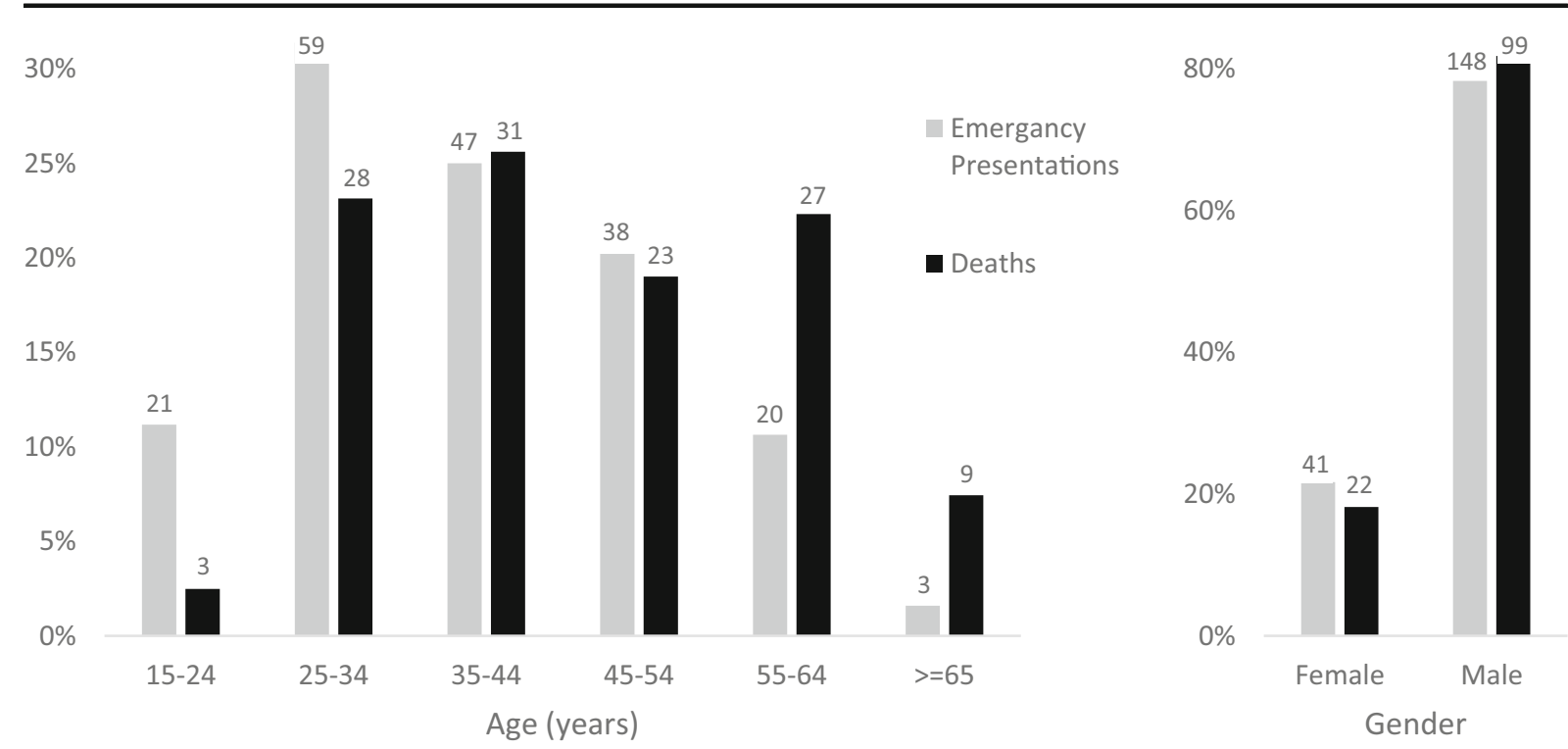

Fig. 1 Gender and ages of individuals who presented to the emergency department or accidentally died due to an opioid overdose from January 1 to April 18, 2020

combination. From March 16 to April 18, there were 1.47 deaths per day, compared with 0.95 deaths per day prior to this period (Fig. 2). In 2020, deaths per day were higher in April than preceding months (Fig. 3). Comparatively, deaths in the months of March and April in 2018 and 2019 were lower than in the month of February in each respective year.

\section{Discussion}

Individuals with substance use disorders, particularly those using opioids, are among vulnerable populations with an increased risk of drug-related harms and death during social distancing regulations $[1,12]$. The delivery of harm reduction measures is difficult during this unprecedented time - persons who use drugs are more likely to use alone, seek out new sources for drugs as supply chains are disrupted, or use unclean supplies given reduced access to needle and syringe exchange programs $[8,9]$. In addition, the risk of death may be increased for users of opioids due to the need for timely reversal treatment such as naloxone. The isolation that comes as a consequence of social distancing may also be a trigger for relapse [12]. Furthermore, reduced access to drug treatment programs and clinics presents as a further risk for relapsing to street-based drugs [13].

The increased number of overdoses seen during our study period may have been related to reduced observations of overdose events as a result of less foot traffic past overdosed individuals who use in public view, or decreased visitation to homes for those using drugs behind closed doors [14]. In addition, reversed overdoses that may not have typically presented to emergency departments and/or medical examiner offices may have become more severe in consequence and required medical and/or death investigation. Based on our results, it appears drug supplies have not been significantly impacted, at least in these initial months of the COVID-19 pandemic. It should also be noted that in recent years, San Francisco has continued to observe increases in fatal overdoses each year, demonstrated when comparing year-to-year total deaths in Fig. 3.

Several limitations exist from this study. First, data collected were only two large emergency departments in the city, but do not reflect all survived overdoses in the city. In addition, emergency department charts were queried for chief complaints and final diagnosis codes, which tend to vary in accuracy and completeness, and therefore, we may have omitted relevant cases or may have been observing an inaccurate, although likely consistent, trend. Furthermore, in most of our emergency department cases, the exact substance(s) contributing to overdose was unknown, resulting in a non-specified opioid chief complaint or final diagnosis. Other limitations include those inherent to cross-sectional studies of this type, such as the inability to establish causation or determine the directionality of relationships. Finally, 


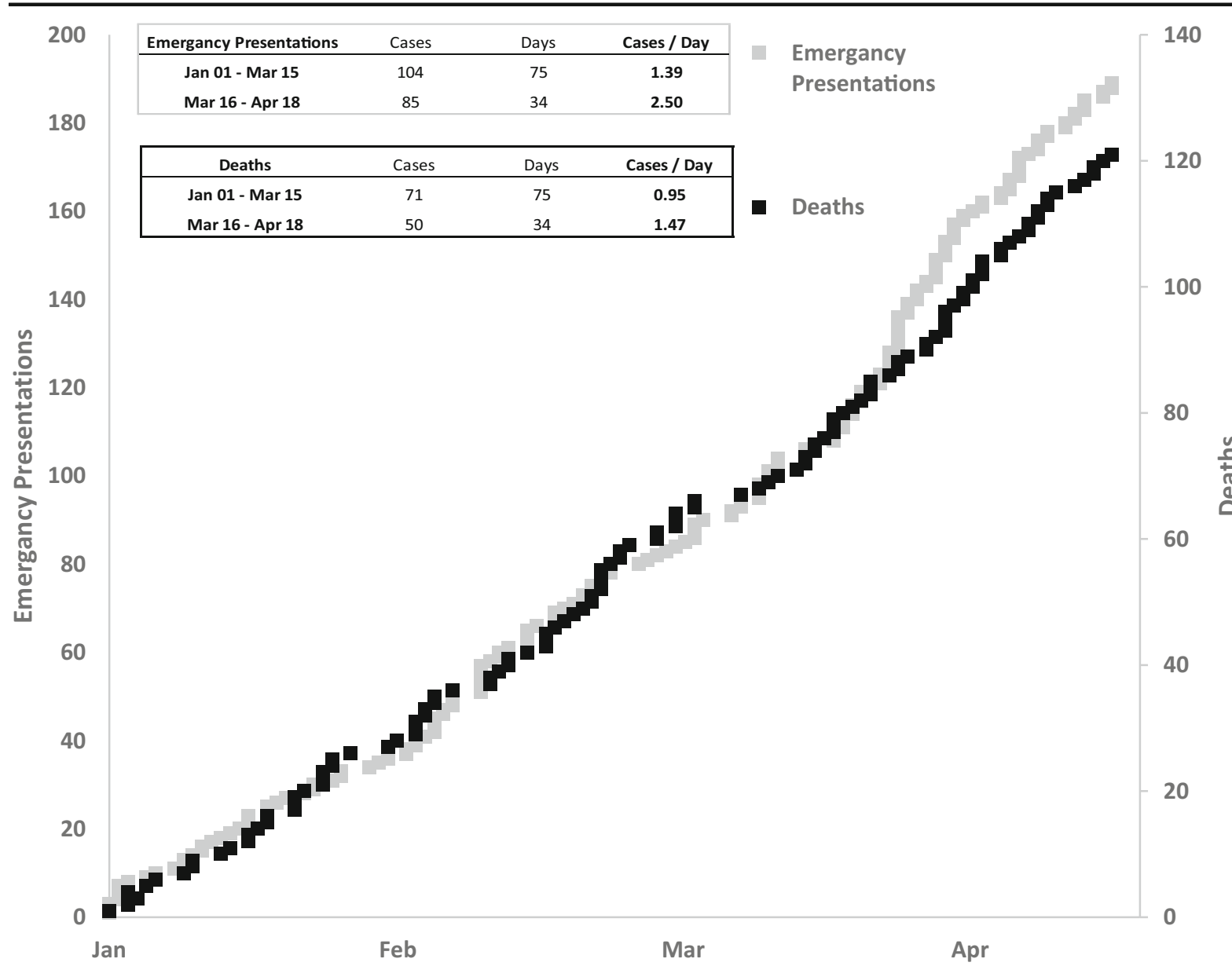

Fig. 2 Emergency department presentations and accidental deaths due to opioid overdose from January 1 to April 18, 2020, following shelter-in-place orders on March 16, 2020

individuals with opioid use disorder may also be particularly susceptible to COVID-19 complications due to substance use-related respiratory problems or hepatic insufficiency and may have affected our number of observed fatalities [14]. Such complications were not considered in this study.

As of April 18, 2020, 665,330 COVID-19positive-confirmed cases were reported, resulting in 30,384 deaths nationwide [15]. At the same time in San Francisco, there were 1215 cases and 20 COVID-19 deaths [16]. The early enactment of protective measures to reduce the rate and amount of COVID-19 spread within a condensed major US city has likely led to the mitigation of thousands of cases and saving of many lives. Considering other major cities, even with the observed increases in drug overdose deaths, there is undoubtedly a total harm minimization. Furthermore, without San Francisco's developed public health measures to reduce drug harm in the community through resources such as widespread naloxone access and user support services, a larger increase in overdoses may have been observed [17]. Finally, San Francisco's comprehensive drug surveillance systems in UCSF and ZSFG emergency rooms, and OCME decedent investigations, may subsequently demonstrate emphasized overdose data relative to other populations with limited analysis, testing, and/or reporting.

The San Francisco Bay Area likely had the first death from COVID-19 back on February 6, 2020, in Santa Clara County [18]. Although the Bay Area was possibly among one of the first locations exposed in the country, early implementation of the aforementioned measures have proven effective to flatten-the-curve, 


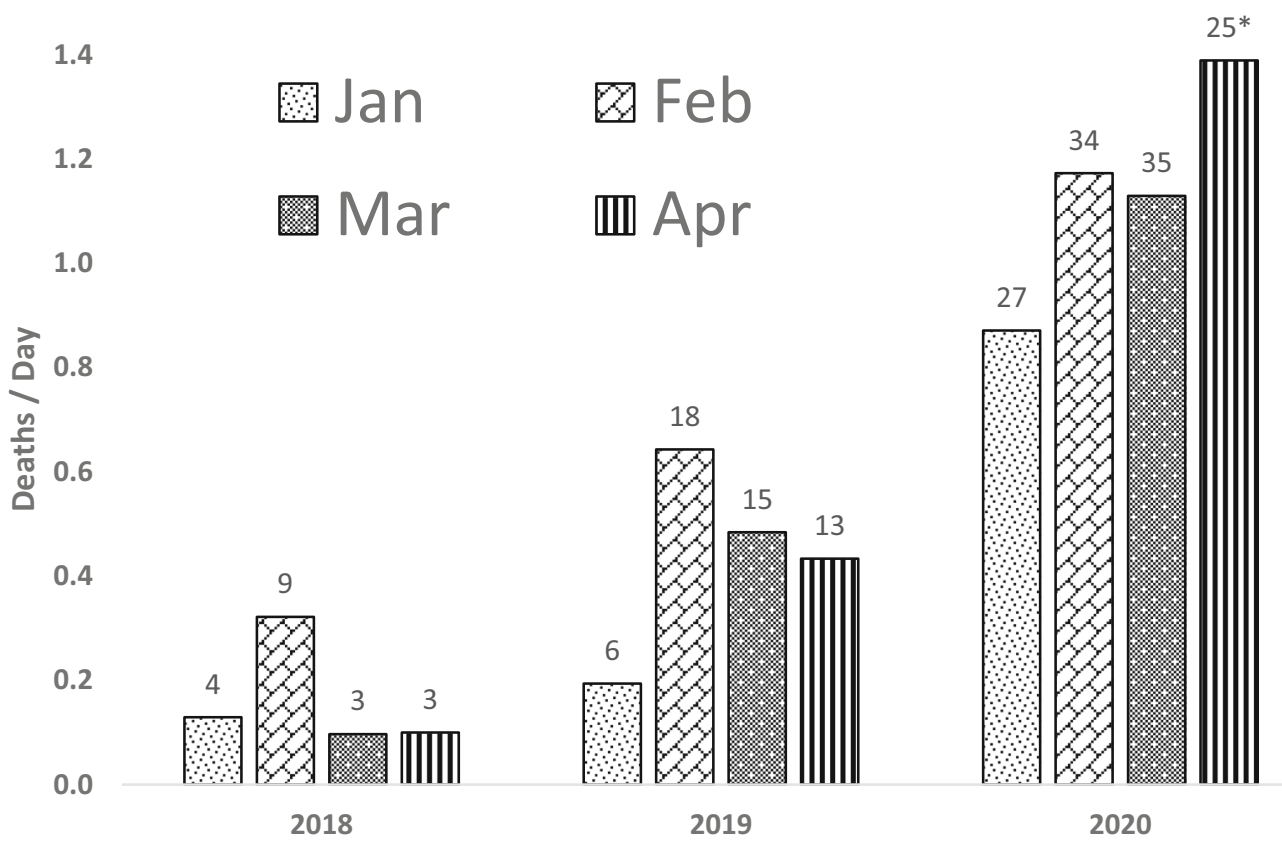

* April 2020 contained cases only up until the study period of April 18.

Fig. 3 Deaths per day and total deaths per month due to opioid overdose from January 1 to April 18, 2020. The asterisk indicates that April 2020 contained cases only up until the study period of April 18

contain the spread of COVID-19, and reduce overwhelming hospitals and, subsequently, deaths from COVID-19.

\section{Conclusion}

Anecdotal commentaries of either drug supply disruption, increased syringe requests, and/or overdoses have been reported in the media. Here, we describe that during the first weeks of a COVID-19 pandemic, emergency room presentations and deaths related to opioid overdose may increase during an isolation period. This report provides useful and immediate information for already strained hospitals, forensic facilities, and public health agencies that come under threat with a simultaneous viral pandemic and drug epidemic.

\section{References}

1. Heimer R, McNeil R, Vlahov D. A community responds to the COVID-19 pandemic: a case study in protecting the health and human rights of people who use drugs. J Urban
Health. 2020;97(4):448-56. https://doi.org/10.1007 /s11524-020-00465-3.

2. Centers for Disease Control and Prevention (CDC). How COVID-19 spreads. Updated April 13, 2020. Accessed April 18, 2020. https://www.cdc.gov/coronavirus/2019ncov/prevent-getting-sick/how-covid-spreads.html

3. World Health Organization (WHO). Coronavirus disease 2019 (COVID-2019) situation report - 72 (April 1, 2020). 2020. April 01, 2020.

4. San Francisco Department of Public Health. Shelter in Place - Order Of The Health Officer No. C19-07. (2020).

5. Executive Department, State of California. Stay at Home Executive-Order-N-33-20. (2020). https://covid19.ca. gov/img/Executive-Order-N-33-20.pdf

6. Ciccarone D, Ondocsin J, Mars SG. Heroin uncertainties: exploring users' perceptions of fentanyl-adulterated and substituted 'heroin. Int J Drug Policy. Aug 2017;46:14655. https://doi.org/10.1016/j.drugpo.2017.06.004.

7. San Francisco Department of Public Health. San Francisco Safe Injection Services Task Force - Final Report. 2017.

8. American College of Medical Toxicology. Mitigating the intersection of COVID-19 and opioid use disorder (Webinar). May 20, 2020.

9. Levander XA, Wakeman SE. Covid-19 will worsen the opioid overdose crisis if we don't prepare now. STAT March 17, 2020

10. Gevorkyan J, Wong M, Pearring S, Rodda LN. Method consolidation to improve scope and efficiency in 
postmortem toxicology. J Anal Toxicol. 2020;44(5):422-39. https://doi.org/10.1093/jat/bkaa003.

11. Stam NC, Gerostamoulos D, Dietze PM, Parsons S, Smith $\mathrm{K}$, Lloyd B, et al. The attribution of a death to heroin: a model to help improve the consistent and transparent classification and reporting of heroin-related deaths. Forensic $S c i$ Int. 2017;281:18-28. https://doi.org/10.1016/j. forsciint.2017.10.012.

12. Becker WC, Fiellin DA. When epidemics collide: coronavirus disease 2019 (COVID-19) and the opioid crisis. Ann Intern Med. 2020:M20-1210. https://doi.org/10.7326/M201210 .

13. National Academies of Sciences E, Medicine,Medications for Opioid Use Disorder Save Lives. The National Academies Press; 2019:174.

14. Volkow ND. Collision of the COVID-19 and addiction epidemics. Ann Intern Med. 2020; https://doi.org/10.7326 /M20-1212.

15. World Health Organization (WHO). Coronavirus Disease 2019 (COVID-2019) Situation report - 89 (April 18, 2020). 2020. April 18, 2020.
16. San Francisco Department of Public Health. Coronavirus (COVID-19) Cases in San Francisco. https://www.sfdph. org/dph/alerts/coronavirus.asp\#. Accessed 28 April 2020.

17. San Francisco Department of Public Health. Harm Reduction Services in San Francisco. (2017).

18. Gafni M, Tucker J. Autopsy report of first known coronavirus death in U.S. says disease caused Santa Clara woman's heart to rupture. San Francisco Chronicle. April 25, 2020. https://www.sfchronicle.com/bayarea/article/ExclusiveAutopsy-report-of-first-known-15226422.php?utm campaign=premiumsfgate_breakingnews_20200425\&utm source $=$ newsletter\&utm_medium $=$ email. $\overline{\text { Accessed }} 28$ April 2020.

Publisher's Note Springer Nature remains neutral with regard to jurisdictional claims in published maps and institutional affiliations. 